\title{
Efficient Wavelet-based Medical Image Fusion Scheme with PCA Fusion Rule
}

\author{
Ahmed Sabeeh Yousif ${ }^{1}$, Zaid Omar ${ }^{1}$, Nur' Aqilah Hamzah ${ }^{1}$
}

\begin{abstract}
Medical image fusion is defined as the retrieval of complementary information from multiple medical images, for diagnostic purposes and clinical analysis. In our research, 2D discrete wavelet transform (2D-DWT) as an image deomposition method, coupled with principal component analysis (PCA) are applied as a new strategy for fusion of medical CT and MRI images. 2D-DWT is an established method of data decomposition and fusion, whereby it is compact and may provide directional information. The novelty of our work is where the decomposed wavelet images are then processed using PCA-based fusion rule. This approach manages to improve upon corners, edges and contrasts of sources image, thereby providing an altogether higher quality fused image. Simulation results demonstrate high visual quality of the fused image supported by higher values of fusion metrics; these justify the effectiveness of the proposed scheme in comparison to previous methods for image fusion.
\end{abstract}

Keywords-CT, MRI, 2D-DWT, PCA, Standard Deviation, Entropy, Average Gradient

\section{Introduction}

In computer vision [1] applications, one of the challenging problems is to merging the relevant information from various images sensors of the same scene without introducing artifacts in the resultant image. Because of the different types of sensors [2,3] are used in image capturing devices and their principle of sensing and also, due to the limited depth of focus of optical lenses used in camera, it is possible to get several images of the same scene providing different information. Therefore, combining different information from several images to get a new improved composite image becomes important area of research. Image fusion applications are found in diverse areas including medical imaging [4-5], forensic science [6], remote sensing [7], and surveillance [7].

The purpose of image fusion is to obtain more accurate, more dependable and more general image by synthesizing several source images. Computer Aided Diagnosis (CAD) is an emerging and big research domain in diagnostic field. Medical imaging technique contributes to create high visual representations of the internal structure details of human body for clinical analysis purpose [8]-[9]. The complementary nature of medical imaging sensors of different modalities, (X-ray, Magnetic Resonance Imaging (MRI), Computed. Tomography (CT)) has brought a great need of image fusion for the retrieval of relevant information from medical images. To elaborate more, CT helps in accessing the extent of disease; yet it is limited in soft-tissue contrast, needed for differentiating tumors from

\footnotetext{
${ }^{1}$ Faculty of Electrical Engineering, Universiti Teknologi Malaysia, 81310
} Skudai, Johor, Malaysia scar tissues. On the other hand, MRI scores over CT in terms of soft tissue discrimination. This is necessary because the soft tissue contrast allows better visualization of tumors. This highlights the need towards the development of multimodality medical imaging sensors for extracting clinical information to explore the possibility of data reduction along with better visual representation [10,11]. Various spatial domain [12,13] and frequency domain $[14,15]$ image fusion methods have been proposed in literature. Some of the popular spatial domain methods are Arithmetic Averaging and Principal Component Analysis (PCA) [15,16], Sharpness criteria [17] and IHS (Intensity Hue Saturation) [18] based fusion schemes.

However, spatial domain image fusion techniques often produce poor because they usually produce edge distortions in the fused image. In the recent years multiresolution approaches using Mallat [28], the à trous [19] transforms, contourlet $[20,21]$ have been proposed for image fusion. Fusion approaches employing wavelets analysis include transforms such as SWT [22], LWT [23], MWT [23], RDWT [24], and complex wavelet [25]. Y. Luo et al. [26] used a PCA plus à trous wavelet transform which based on the spatial and spectral resolutions. But, the technique did not get emphasis on edge or shape detection, which are fundamental structures in natural images and particularly relevant from a visual point of view. Y. Yang et al. [27] proposed a fusion method based on window selection and the discrete wavelet transform. The technique emphasized on the treatment of the low and high frequency bands with different selection rule separately. The method proposed performed better than pixel averaging and conventional DWT with maximum selection rule, but has a limitation of less contrast in the fused image. D. A. Godse in their work [28] selected maximum pixel intensity approach along with wavelet to perform fusion. The said combination produced a focused image but the image suffered with blurring. On the other hand, work of R. Singh and A. Khare [29] conferred a method integrating Daubechies complex wavelet transform and weighted average rule for fusion but resulting in a highly blurred image. As, both the relevant and irrelevant information from the source images are included in the fused one. S. K. Sadhasivam in their work [30] applied PCA along with the selection of maximum pixel intensity to perform fusion. The method yielded an image with less structural similarity with the source images along with low contrast and luminescence. The reason behind choosing twodimensional discrete wavelet transform (2D-DWT) is more compact, and able to provide directional information in the low-low, high-low, low-high, and high high bands, and contains unique information at different resolutions [31]. Thereby yielding a suitable approach for medical image fusion. The proposed work therefore presents a combination of two-dimensional discrete wavelet transform (2D-DWT) and PCA (Principal Component Analysis) as an 
Proc. of the Fourth Intl. Conf. Advances in Computing, Communication and Information Technology- CCIT 2016 Copyright (C) Institute of Research Engineers and Doctors, USA .All rights reserved. ISBN: 978-1-63248-092-7 doi: 10.15224/ 978-1-63248-092-7-45

improvement to the aforementioned limitations. The obtained results have been evaluated using entropy (E), standard deviation and average gradient.

\section{Two-dimensional Discrete Wavelet Transform}

The wavelet transform was applied to image processing successfully, which was first discovered and developed by S. Mallat [32]. The 2-D DWT can be seen as a 1-D wavelet transform along the row and column direction, and can be computed with a separable extension of the 1-D decomposition algorithm. A multiresolution representation provides a simple hierarchical framework for interpreting the image information. At different resolutions, the details of an image generally characterize different physical structures of the scene. The 2-D DWT can be implemented by applying the two channel decomposition of I-D DWT in the horizontal and vertical dimensions [33]. The scaling and wavelet coefficients for the 2-D DWT are given:

$$
\begin{aligned}
& C_{j+1 \mathrm{mn}}=\sum_{\mathrm{i}=-\mathrm{s}}^{\mathrm{m}} \sum_{\mathrm{k}=-\mathrm{m}}^{\mathrm{m}} h(i-2 m) h(\mathrm{k}-2 \mathrm{n}) \mathrm{C}_{j \tilde{\mathrm{I}}}
\end{aligned}
$$

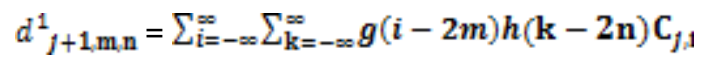

$$
\begin{aligned}
& d_{f+1 \mathrm{mn}}^{2}=\sum_{\tilde{i}=-\mathrm{s}}^{\mathrm{s}} \sum_{\mathrm{k}=-\mathrm{se}}^{\mathrm{m}} h(i-2 m) g(\mathbf{k}-\mathbf{2 n}) \mathrm{C}_{j \mathrm{j}}
\end{aligned}
$$$$
\text { (1) }
$$

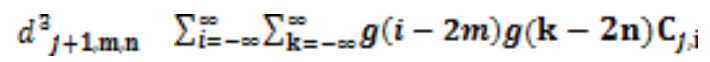

Where $C_{j+1 \text { mnn }}$ represent the lower half of frequency range at position ( $\left.2^{J_{m}} 2^{l_{n}}\right)$ and $d_{j+1, m n}^{1}, d^{2}{ }_{j+1, m n} d^{a}{ }_{j+1, m n}$ represent the upper half of the frequency range in the vertical, horizontal and diagonal direction.

\section{A. General block diagram of propose approach}

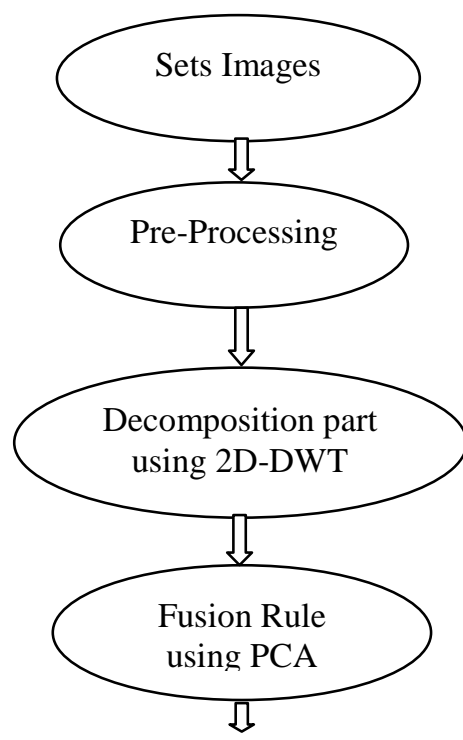

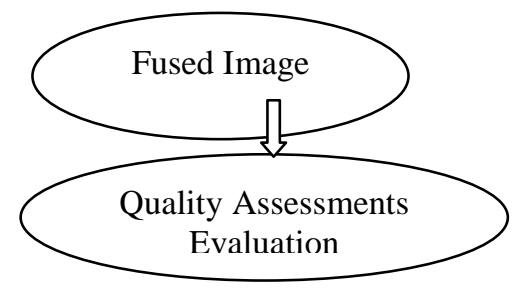

Fig. 1: Block Diagram of Procedure Involved in Proposed Fusion Algorithm.

\section{B. Proposed Fusion Method}

Pre-processing is the first step that include preamble the source images that used as database and convert the input images from RGB scale to gray scale in the test of CT and MRI images but in case of used noise image we will use normal gray scale image as database, Second 2D-DWT will play a role for decomposed the two sources images into four level, in this case only one level applied because our research focused on single level's sub-bands, frequency bands namely a lower-frequency band and other higherfrequency bands. 2D-DWT are able to provide directional information in the low-low, high-low, low-high, and highhigh bands, and contains unique information at different resolutions and that which makes fusion process better by merging the wavelet coefficients. In the next step after obtained the approximation and coefficients details, PCA is applied as a fusion rule to selectively merging the proper wavelet coefficients of input images. PCA contributes for convert features from original domain to a PCA domain. PCA domain bring a big amount of information This can be achieved by retaining only those components which have a larger variance and PCA proposed algorithm shown in fig. 2; The next step involves, the reconstruction of the processed coefficients (after PCA fusion rule) using inverse DWT transform to generate the fused image.

\section{Wavelet coefficients of inputs images}

Calculate the column vectors of wavelet coefficients

By using these vectors above, calculate Covariance matrix

Process: Diagonal elements of the covariance vector.

Compute: Eigen vectors and Eigen values of covariance

Divide each element with the mean of Eigen Vector by process Column vector corresponding to large Eigen value 
Proc. of the Fourth Intl. Conf. Advances in Computing, Communication and Information Technology- CCIT 2016

Copyright (C) Institute of Research Engineers and Doctors, USA .All rights reserved.

ISBN: 978-1-63248-092-7 doi: 10.15224/ 978-1-63248-092-7-45

Result $=($ normalized Eigen vector values $)(X)($ term of complex wavelet coefficient matrix. and repeat for

Compute: Inverse wavelet transform of scaled matrices.

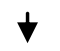

Fused image equation $=$ summation of above matrices

Fig. 2: PCA Algorithm in Wavelet Domain.

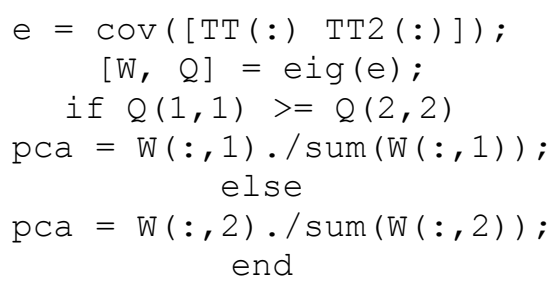

Fig. 3: Pseudo code of PCA based on Matlab programming

\section{Image Quality assessments}

After obtained fused images, we choose some performance criteria, entropy, average gradient, standard deviation and to measure the quality of the fused images. In general, larger entropy and average gradient indicate that the fused image contains more information. Larger average gradient indicates the fused image contains more details and texture feature.

\section{1) Standard Deviation $(\sigma)$}

Standard deviation is the measure of the contrast of the fused image and it can be calculated as:

$$
\sigma=\sqrt{\sum_{I=0}^{L-1}(i-\bar{i})} h_{F}(i), \bar{i}=\sum_{i=0}^{L-1} i h_{F}(i)
$$

where $i, h_{F}$ and $L$ are the grey-level index, the normalized histogram of the fused image, and the number of bins in histogram, respectively. Higher the value of standard deviation better is the quality fused image.

\section{2) Entropy}

Entropy of an image is the measure of the information content in the fused image. Higher values of entropy indicate that the fused image contains more information than the other fused images. Entropy is given by Eq. (2).

$$
E=-\sum_{L=0}^{L-1} P_{1} \log _{2} P_{1}
$$

Where: $\mathbb{L}$ represents no. of gray level, $\mathbb{R}_{\mathbb{1}}$ is the ratio between the no. of pixels with gray values / and total no. of pixels.

\section{3) Average gradient}

For the spatial quality, we use the average gradient to evaluate the performance of the fused image $\mathrm{F}$. That is

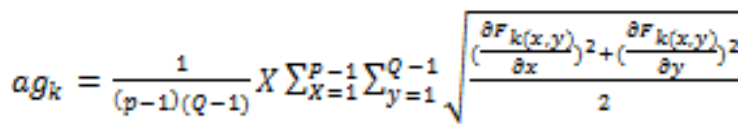

$$
\begin{aligned}
& k=R_{x} G_{x} B
\end{aligned}
$$

where $F_{k}(\mathrm{x}, \mathrm{y}) \mathrm{k}$ is the pixel value of the fused image at position $(\mathrm{x}, \mathrm{y})$. The average gradient reflects the clarity of the fused image. It can be used to measure the spatial resolution of the fused image, i.e., a larger average gradient means a higher spatial resolution.

\section{Experimental results}

In this section, the experimental results have obtained using various sets of images. CT and MRI medical images have been tested with $(256 \times 256)$ pixel and also images are artificial blurred and noise $(512 \times 512)$ pixel. The fusion quality measure has been used to evaluate the fusion approach. The results of fused images obtained using the proposed approach are given $n$ Fig. 3; while, the quantitative analysis of the same has been shown in table 1. In the fig. 3 fused image has high quality and that will give advantage in the diagnostic field and be more reliable to the clinical analysis. This is supported by high values of fusion metrics that applied (Entropy ,Standard deviation, average gradient) and can see also fused image contain complementary information of both images in case of (CT,MRI) and (image noisy-blur with original image). All this advantage can led to determine the extent disease. From results in Fig. 3, we can easily conclude that fused image obtained by the proposed method provides details of boney structures as well as information about soft tissue.
Set-1

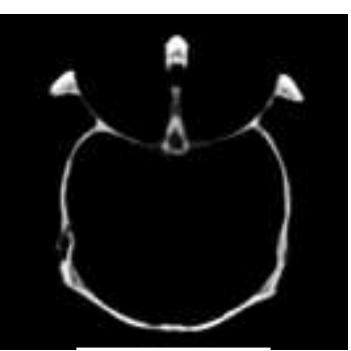

A

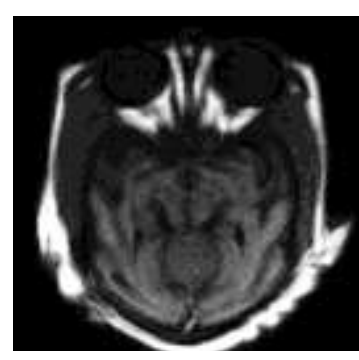

Set-2

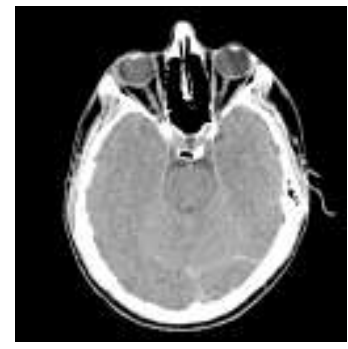

A1

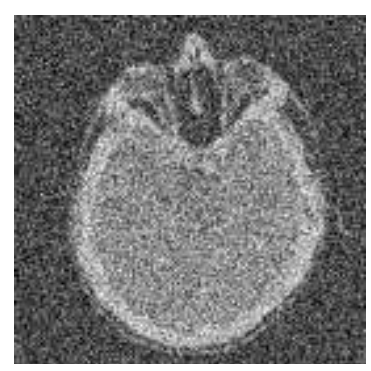


Proc. of the Fourth Intl. Conf. Advances in Computing, Communication and Information Technology- CCIT 2016 Copyright (C) Institute of Research Engineers and Doctors, USA .All rights reserved.

ISBN: 978-1-63248-092-7 doi: 10.15224/ 978-1-63248-092-7-45

B

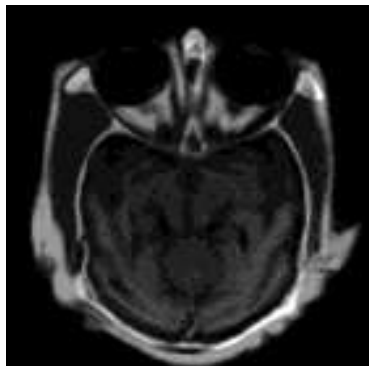

Fig. 4: Image Fusion Results with the Proposed Approach of different sets. (A) \& (B) Input CT and MR images, (A1) Input CT image, (B2) Input CT image with noise and blur, (C) \& (C1) fused images.

Table 1: Quantitative Analysis of Proposed Fusion

method.
\begin{tabular}{|l|l|l|l|}
\hline Set No. & Entropy & $\begin{array}{l}\text { Standard } \\
\text { Deviation }\end{array}$ & $\begin{array}{l}\text { Average } \\
\text { Gradient }\end{array}$ \\
\hline Set-1 & 6.15 & 41.8 & 4.3 \\
\hline Set-2 & 6.2 & 38.2 & 11.6 \\
\hline
\end{tabular}

From our proposed algorithm, it clear that in this case the fused image obtained by the proposed method is more informative and can see that for the soft tissue appear in fused image in Fig.3 (C1), after CT input image blurred, soft tissue will remove from left side, in the fused image will see it perfectly and that give to the proposed method better point in the diagnose analysis. To compare our proposed algorithm with state of arts of fusion methods, we used set-1 to compare with other methods (PCA fusion, linear fusion, DWT based fusion and LWT based fusion and Daubechies Complex Wavelet Transform). The comparison divided in two parts:

\section{1) Visual representation}

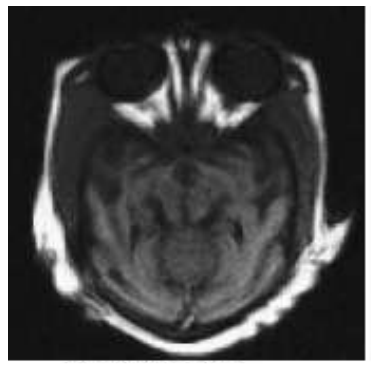

(a).MRI image .

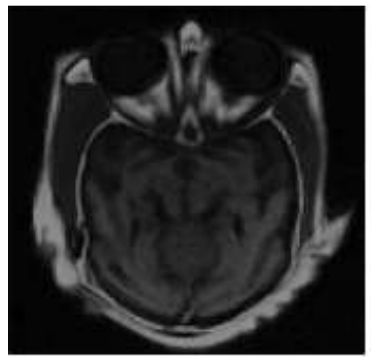

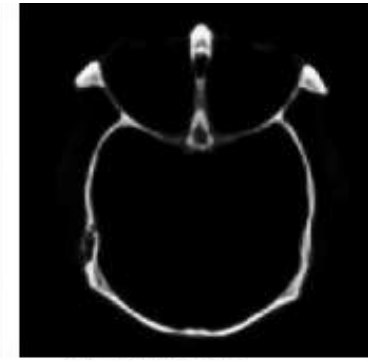

(b).CT image.

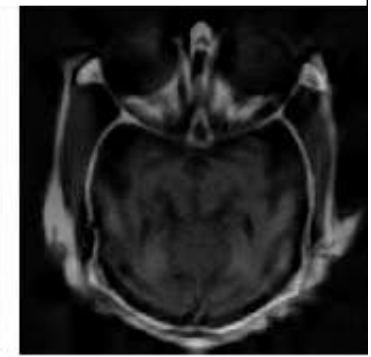

(c) Complex Wavelet Transform. (d).LWT based fusion.

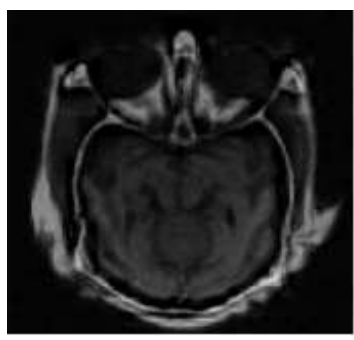

(e). DWT based fusion [41].

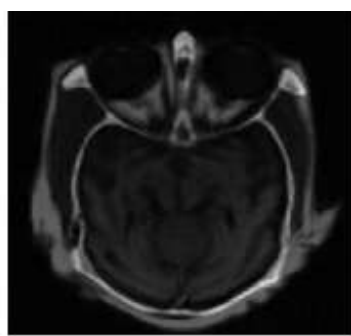

(f). Linear fusion 「40].

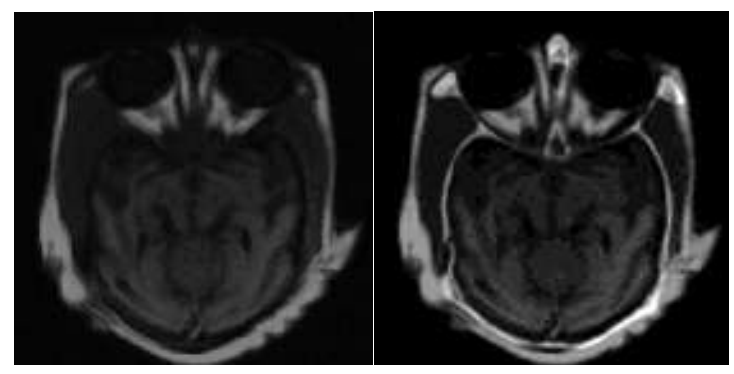

(g).PCA fusion [39].

(q). proposed fusion method

Fig. 5: Comparison of fusion algorithms of first sets with proposed fusion method in term of visual representation.

The visual representation in the Fig. 4; shown better view of fusion method proposed than other in term of (edge, soft tissue, shape) but is not sufficient for analysis of the obtained fusion results. Therefore we have compared the proposed method with other ones on quantitative measures.

\section{2) Quality assessment metrics}

In this section state of arts that done before used with same databases, so the proposed algorithm will compare with (PCA fusion, linear fusion, DWT based fusion and LWT based fusion and Daubechies Complex Wavelet Transform) and that given in the Table 2.

Table 2: Performance Comparison for first set of medical images

\begin{tabular}{|c|c|c|}
\hline Algorithms & Entropy & $\begin{array}{c}\text { Standard } \\
\text { Deviation }\end{array}$ \\
\hline $\begin{array}{c}\text { DWT based } \\
\text { fusion }\end{array}$ & 6.0640 & 33.3744 \\
\hline Linear fusion & 5.6557 & 29.6860 \\
\hline $\begin{array}{c}\text { LWT based } \\
\text { fusion }\end{array}$ & 6.1921 & 34.0205 \\
\hline $\begin{array}{c}\text { Complex Wavelet } \\
\text { Transform }\end{array}$ & 6.0013 & 32.8511 \\
\hline PCA fusion & 5.7638 & 28.3914 \\
\hline $\begin{array}{c}\text { Proposed } \\
\text { method }\end{array}$ & $\mathbf{6 . 1 5}$ & $\mathbf{4 1 . 2 5 0 9}$ \\
\hline
\end{tabular}


By considering the ranking of methods on each measure, it can be concluded that the proposed method excels majority of other methods in every performance measure.

\section{Conclusions}

The retrieval of complementary information from different sensors of medical imaging is an important issue in the clinical diagnosis and analysis. In this paper, we have proposed a fusion method based on 2D-DWT with principal component analysis (PCA). This fusion scheme is helpful in obtaining clear image features and details such as edges, shapes, and corners. Performance evaluation of the obtained results are done with several well-defined fusion measures i.e. entropy, standard deviation and average gradient. The proposed fusion is found to be more refined in its visual representation as well as able to highlight soft tissue details. On the basis of quality assessments metrics and visual analysis, we can affirm the proposed fusion method to be better than other fusion approaches, be it transform domain methods (LWT and regular DWT) or spatial domain methods (PCA and linear fusion).

\section{Acknowledgement}

We would like to thank Universiti Teknologi Malaysia for kindly sponsoring this research under the Tier 1 Research University Grant (vote no. 12H72).

\section{REFERENCES}

[1] D. A. Forsyth and J. Ponce, "Computer Vision - A Modern Approach", PHI Publication, 2009

[2] R. S. Blum and Z. Liu., "Multi-sensor image fusion and its applications", CRC Press, Taylor \& Francis Group, 2006.

[3] Yi Chai, Huafeng Li and Zhaofei Li, "Multifocus image fusion scheme using focused region detection and multiresolution," Optics Communications, Vol. 284, pp. 4376-4389, 2011.

[4] R. Singh, R. Srivastava, O. Prakash and A. Khare, "Mixed scheme based multimodal medical image fusion using Daubechies complex wavelet transform," in proc. of IEEE/OSA/IAPR International conference on Informatics, Electronics and Vision, Dhaka, pp. 304309, 2012.

[5] B. V. Darasthy, "Information fusion in the realm of medical applications - abibliographic glimpse at its growing appeal", Information Fusion Vol. 13 pp. 1-9, 2012.

[6] C.Y. Wen, J.K. Chen, "Multi-resolution image fusion technique and its application to forensic science", Forensic Science International, vol.140, pp.217-232, 2004

[7] P. Shah, S. N.Merchant and U. B.Desai, "Fusion of surveillance Images in Infrared and visible band using curvelet, wavelet and wavelet packet transform ,"International Journal of Wavelets, Multiresolution and Information Processing Vol. 8, No. 2, pp. 271$292,2010$.

[8] El-Dahshan, El-Sayed A., Heba M. Mohsen, Kenneth Revett, and Abdel-Badeeh M. Salem. "Computer-aided diagnosis of human brain tumor through MRI: A survey and a new algorithm." Expert Systems with Applications 41, no. 11 (2014): 5526-5545.

[9] Lay-Ekuakille, Aimé, Patrizia Vergallo, D. Stefano, Alessandro Massaro, Antonio Trabacca, Matteo Cacciola, Domenico Labate, Francesco Carlo Morabito, and Rosario Morello. "Diffusion tensor imaging measurements for neuro-detection." In Medical Measurements and Applications Proceedings (MeMeA), 2012 IEEE International Symposium on, pp. 1-4. IEEE, 2012.

[10] Soloperto, Giulia, Francesco Conversano, Ernesto Casciaro, Antonio Greco, Giuseppe Gigli, Aime Lay-Ekuakille, and Sergio Casciaro. "Laser fluence and exposure time effects on optoacoustic signal from gold nanorods for enhanced medical imaging." In Instrumentation and Measurement Technology Conference (I2MTC) Proceedings, 2014 IEEE International, pp. 1139-1143. IEEE, 2014.

[11] Lay-Ekuakille, A., G. Vendramin, A. Trotta, I. Sgura, T. Zielinski, and
P. Turcza. "Accuracy assessment of sensed biomedical images for myocardial infarction prediction." In Sensing Technology, 2008. ICST 2008. 3rd International Conference on, pp. 457-461. IEEE, 2008.

[12] P. Shah, S. N.Merchant and U. B.Desai, "An efficient spatial domain fusion scheme for multifocus images using statistical properties of neighborhood," Multimedia and Expo (ICME), pp. 1-6, 2011.

[13] H. Chen, "A multiresolution Image Fusion based on Principle Component Analysis," Fourth International Conference on Image and Graphics, pp. 737-741, 2007.

[14] H. H. Wang, "A new multiwavelet-based approach to image fusion", Journal of Mathematical Imaging and Vision, vol.21, pp.177$192,2004$.

[15] R. Singh, R. Srivastava, O. Prakash and A. Khare, "DTCWT based multimodal medical image fusion", in proc. of International conference on Signal, Image and Video processing, January 2012, pp. 403-407, IIT Patna.

[16] V. P. S. Naidu and J. R. Raol, Pixel-level image fusion using wavelets and principal component analysis", Defence Science Journal, Vol. 58,

No. 3, pp. 338-352, 2008

[17] J. Tian, L. Chen, L. Ma and W. Yu, "Multi-focus image fusion using a bilateral gradient-based sharpness criterion," Optics Communications, Vol. 284, pp. 80-87, 2011.

[18] S. Daneshvar and H. Ghassemian, "MRI and PET image fusion by combining IHS and retina-inspired models", Information Fusion Vol. 11, No. 2, pp. 114-123, 2010.

[19] M. J. Shensa, "The discrete wavelet transform: Wedding the à Trous and Mallat algorithms," IEEE Trans. Signal Process., Vol. 40, No. 10, pp. 2464-2482, Oct. 1992

[20] M. Alejaily Aboubaker, "Fusion of Remote Sensing Images Using Contourlet Transform. Innovations and Advanced Techniques in Systems," Computing Sciences and Software Engineering, Springer, pp. 213-218, 2008 .

[21] L.D Cunha, J.P Zhou, "The Non-Subsampled Contourlet Transform: Theory, Design, and Applications," IEEE Transactions on Image Processing, Vol. 15, No. 10, pp. 3089-3101, 2006.

[22] P. B. Watanadelok, W. Rattanapitak, S. Udomhunsakul, "Multi-focus image Fusion based on Stationary Wavelet Transform and extended spatial frequency measurement," IEEE Transactions on Image Processing, pp. 7781,2009

[23] K. Kannan, S. A. Perumal and K. Arulmozhi, "Performance Comparison

of Various Levels of Fusion of Multi-focused Images using Wavelet Transform," International Journal of Computer Applications, Vol. 1, No. 6, pp. 71-78, 2010.

[24] R. Singh, M. Vatsa, A. Noore, "Multimodal Medical Medical Image Fusion using Redundant Wavelet Transform," in the proceeding of Seventh International Conference on Advances in Pattern Recognition, pp. 232-235, 2009.

[25] R. Singh and A. Khare, "Fusion of Multimodal Images using Daubechies Complex Wavelet Transform- A Multiresolution Approach," Elsevier-Information Fusion, Vol. 19, pp. 49-60, 2014.

[26] Y. Luo, R. Liu and Y. Z. Zhu, "Fusion of Remote Sensing Image Based on the PCA + a trous Wavelet Transform," The International Archives

of the Photogrammetry, Remote Sensing and Spatial Information Sciences, Vol. XXXVII, Part B7, pp. 1155-1158, Beijing (China),2008

[27] Y. Yang, D. S. Park, S. Huang and N. Rao, "Medical Image Fusion via an Effective Wavelet-Based Approach," EURASIP Journal on Advances in Signal Processing, Article ID 579341, pp. 1-13, March 2010

[28] D. A. Godse and D. S. Bormane, "Wavelet based Image Fusion using Pixel based Maximum Selection rule," International Journal of Engineering Science and Technology, Vol. 3, No. 7, pp. 5572-5578, 2011.

[29] R. Singh and A. Khare, " Multimodal Medical Image Fusion using Daubechies Complex Wavelet Transform," IEEE Conference on Information and Communication Technologies, Jeju Islands, South Korea, pp. 869-73, April 2013.

[30] S. K. Sadhasivam, M. K. Keerthivasan and Muttan S, "Implementation of Max Principle with PCA in Image Fusion for Surveillance and Navigation Application," Electronic Letters on Computer Vision and Image Analysis, Vol. 10, No. 1, pp. 1-10, 2011.

[31]MALLAT, S.G.: 'A theory for multiresolution signal decomposition: The wavelet representation', IEEE Trans. Pattern And Mtrch. Intell., 1989, 11, (7), pp. 674-693

[32] S. G. Mallat, "A theory of multiresolution signal decomposition: the wavelet representation," IEEE Trans on Pattern Analysis and Machine Intelligence, vol. 11, July 1989, pp. 674-693. 